Décembre 2002

\title{
Les essaimages académiques dans le secteur de l'informatique en France : Effets institutionnels, effets de territoire ou construction des acteurs locaux?
}

\author{
Caroline Lanciano-Morandat* et Hiroatsu Nohara* \\ * Laboratory of Labour Economics and Industrial Sociology, (LEST-CNRS), \\ Aix-Marseille University, Aix-en-Provence, France
}

Article publié dans la Revue d'Economie Régionale et Urbaine $n^{\circ}$ 2. 2003. pp 235-266.

\section{Résumé}

Cet article s'interroge sur les déterminants des essaimages issus de la recherche publique. Il s'agit de comprendre dans quelle mesure la création de sociétés technologiques issues du système académique dépend d'effets institutionnels, des dynamiques territoriales ou des savoirs et des expertises produits par/encastrés dans les acteurs locaux. Cette analyse est effectuée à partir de l'étude des essaimages issus d'une même politique de transfert des résultats de la recherche au niveau national - celle de l'Institut de Recherche en Informatique et en Automatique (INRIA) - appliquée au même secteur de l'informatique mais à des environnements locaux différents : celui du département de l'Isère ; celui de la technopôle de Sophia Antipolis.

Abstract

This article aims to question the conditions of academic spin-offs companies emerging from the French public research. We attempt to assess to what extent the creation of high tech companies in connection with academic research depends on three following factors: institutional effect associated with the public research, territorial or regional dynamic effect and local actor effect. This analysis is based on an empirical research on the spin-off creation policy of INRIA (National Institute for Computer Science). The main analytical target is to compare the ways in which the same framework of knowledge transfer policy in INRIA can be implemented differently, according to the particular regional dynamics of Grenoble and Sophia Antipolis, or the nature of competence/social network the local actors construct in these two sites.

Mots-clés: Essaimage académique, secteur informatique, institution, territoire, acteurs locaux.

Key-Words : Academic spin-offs, comùputer sector, instituion, region, local actors.

Classification JEL : R58, L14, L86, M13. 
L'objet de cet article $^{1}$ est de s'interroger sur les déterminants de la création de «sociétés technologiques» issues de la recherche publique. Qui des politiques publiques nationales, des dynamiques territoriales ou des savoirs et des savoir-faire produits dans les collectifs locaux agissent sur la création de ces entreprises ? Cette interrogation s'effectue à partir de la comparaison entre des essaimages issus d'une même institution de recherche l'Institut de Recherche en Informatique et en Automatique, (Inria) - dans deux «territoires » différents, le département de l'Isère et la technopôle de Sophia Antipolis.

Les sociétés technologiques issues de la recherche publique (les essaimages ${ }^{3}$ ou «jeunes pousses ») sont analysées dans cet article comme des acteurs d'intermédiation, parmi d'autres, entre trois espaces - l'espace d'enseignement supérieur et de recherche, l'espace industriel et l'espace public - qui génèrent ensemble la dynamique de l'innovation. Alors que l'entreprise et l'espace industriel occupaient la position centrale dans cette dynamique (Lanciano, Maurice, Nohara, Silvestre 1998), ils s'effacent, au début des années 2000, au profit de l'interaction entre ces trois espaces. Cela ne veut pas dire que l'entreprise ait perdu définitivement de son importance dans les dispositifs de l'innovation. Simplement, il devient de moins en moins probable qu'elle puisse exister, sans incorporer les ressources ou compétences externes ou les co-produire avec d'autres. Les frontières de l'entreprise deviennent floues, perméables ou malléables, en même temps que les alliances se multiplient. Les institutions d'enseignement supérieur et de recherche n'ont plus seulement un rôle de producteur des diplômés et des connaissances mais aussi celui d'acteur économique, ce qui se traduit par l'émergence d'un esprit entrepreneurial dans les universités (Ezkovitz et ii 2000 b). L'Etat s'ouvre sur différents acteurs publics (collectifs divers, institutions...) (Verdier 1999) caractérisés plus par leur production de biens publics à différents niveaux (local, régional...) que par leur participation aux actes régaliens du gouvernement. Les relations entre ces trois espaces et entre leurs acteurs respectifs ne se font pas toujours directement et nécessitent parfois l'intermédiation de nouveaux acteurs. Les essaimages sont de ces acteurs.

Cet article se situe dans la problématique esquissée par les théoriciens de la «triple Hélice » (Erzkovitz, Leydesdorf 2000 ), lorsqu'ils s'interrogent sur les mécanismes de transfert de connaissances. Comme chacune des trois hélices, chacun de nos espaces représentant un de ces systèmes possède une cohérence interne, une dynamique, une stratégie, une capacité de changement. A chaque mise en relation entre ces différents partenaires, l'interaction entre les diverses cohérences et dynamiques suscite des réactions variées, non homogènes et non synchronisées qui agissent et qui bousculent les logiques internes des partenaires. Ce mouvement de réajustements mutuels et permanents oblige chacun d'entre eux à négocier et à effectuer des «arrangements » successifs en interne et vis-à-vis du partenariat et contribue ainsi à construire et à reconstruire un nouvel espace dit «intermédiaire ». Mais ces espaces, contrairement à la triple hélice, reposent sur la notion de construction d'acteurs. Les acteurs, conçus par l'analyse sociétale, ne sont pas les agents de la théorie économique,

\footnotetext{
${ }^{1}$ La recherche qui supporte cet article a été menée au sein du projet «Système d'enseignement supérieur et de recherche et système d'innovation des entreprises» (SESI) financé par la Communauté européenne (TSER SOE1-CT97-1054)

${ }^{2}$ Nous reprenons la notion telle qu'elle est présentée dans Colletis, Gilly, Pecqueur 2001, c'est-à-dire comme « une construction sociale d'un groupe composite, structurée autour de ressources partagées (souvent insérée dans un espace physique structuré) ou de l'élaboration d'un avantage relatif ».

${ }^{3}$ La notion d' «essaimage »est considérée ici comme la traduction littérale du terme anglo-saxon, spin-off. Elle recouvre des créations d'entreprises (les jeunes pousses ou start-up), qui ont comme support une innovation technologique et qui sont issues d'une entreprise ou d'une institution publique. Elle est plus large que «la création d'entreprise par un chercheur public » (Mustar 1995) qui implique que le créateur soit un chercheur titulaire (statut) d'une institution de recherche publique.
} 
ni des sujets individuels, ni même des acteurs uniquement stratégiques de Crozier et Friedberg. Cette notion «traduit toute entité individuelle ou collective ayant une capacité de socialisation ou de structuration.» (Maurice, Sellier, Silvestre 1982). Elle peut donc s'appliquer aussi bien à des individus qu'à des collectifs comme les groupes professionnels. Les acteurs ont à la fois une profondeur historique et une réactivité par rapport à leur environnement qui conditionnent leurs pratiques et une possibilité d'influer sur celles-ci en fonction de leurs stratégies immédiates ou à long terme. Ceci nous amène à les distinguer, contrairement aux chercheurs du CSI (Callon, Law 1995), des supports d'activités que sont les outils de travail, les instruments, les équipements techniques et des supports de communication ou de valorisation, par exemple les publications et les brevets. La tension qu'ils subissent entre détermination et autonomie est à la fois à l'origine de la stabilité des logiques d'action, et de leur dynamique, c'est-à-dire de leur capacité à évoluer. Ces logiques se déploient dans le cadre de l'espace intermédiaire entre les entreprises, les institutions d'enseignement supérieur et de recherche et les acteurs publics.

A l'intérieur de ce cadre théorique, nous analyserons dans une première partie les huit essaimages et les différences observées entre ceux de Sophia Antipolis et ceux de Grenoble, puis dans une deuxième partie les déterminants (institution, système territorial d'innovation, pratiques locales des acteurs) des créations de sociétés technologiques, pour essayer de comprendre comment ces différents déterminants interagissent sur l'espace intermédiaire et donc sur la création d'essaimages, dans une troisième partie. Compte tenu du nombre limité des cas observés et de leur état évolutif, il ne s'agit pas ici d'évaluer l'efficience des dispositifs de création d'essaimages à l'aune de quelques indicateurs, mais d'éclairer la nature de ces «output/essaimages » et le processus de leur création, en déconstruisant deux types différents de cohérence locale engendrés par l'interaction entre logique institutionnelle nationale et logique territoriale.

L'Inria est appréhendé comme une institution en tant qu' "infrastructure institutionnelle » (Nelson 1988, 1993), c'est-à-dire comme une structure formelle poursuivant un objectif explicite de politique publique nationale (2-1) qui est, dans le cas présent, le transfert des savoirs et savoir-faire produits par la recherche publique.

L'implantation de ces essaimages sur deux sites géographiques différents nous conduit à nous interroger sur la part de l'effet territorial dans l'émergence de ces entreprises (2-2). Nous utiliserons principalement, pour comprendre ce phénomène, les travaux du groupe «Dynamique de Proximité » (Gilly et Grossetti 1993, Grossetti 1995, Colletis, Gilly, Pecqueur 2001).

Les systèmes d'innovation localisés influent comme le dispositif public national sur les règles, les lois, les pratiques et les routines qui régulent les relations et les interactions au sein des collectifs locaux de recherche (Edqusit,1997). Ainsi, les pratiques des chercheurs et des ingénieurs de l'Inria dans chacun des territoires ne sont pas naturellement identiques et les savoirs et les savoir-faire construits dans chacune des unités de recherche territoriales peuvent faciliter ou non différemment la création d'entreprise (2-3).

Cette analyse des essaimages et de leurs déterminants, basée sur une étude qualitative, doit nous aider à comprendre comment chacun de ces différents contextes au niveau national (institution), territorial (le site) et local (les équipes de recherche) interagit pour permettre ces créations.

\section{I- Les essaimages de l'Inria :}

Le processus de création de «sociétés technologiques à partir de l'Inria » s'engage en 1984 pour faire face à des situations exceptionnelles qui conjuguent la rencontre entre « une 
technologie d'avant-garde pour laquelle le rôle de la recherche est primordiale », un nouveau marché et l'absence d'un industriel concerné. Depuis cette date, l'Inria a été à l'origine de 49 sociétés dont 36 sont toujours en activité sous leur nom propre et 13 ont fusionné avec d'autres entreprises ou ont cessé leur activité ${ }^{1}$.

\section{Courbe des créations}

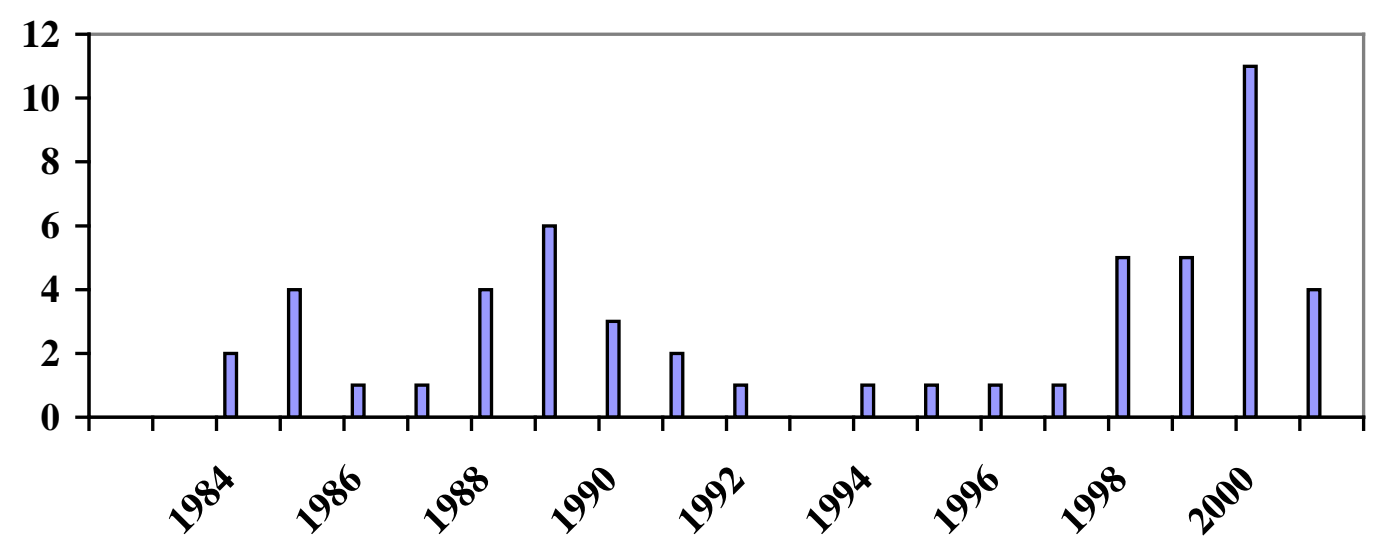

La courbe des créations met en évidence certaines années plus «productives » en sociétés technologiques que d'autres. Ainsi, les sociétés créées dans les années 1988-1989 (premier pic) ont, semble-t-il, utilisé surtout l'émergence d'un besoin du marché en logicielimage et des compétences réseau et de calculateurs temps réel de l'Inria. Mais ces années là ont vu aussi la disparition de 5 sociétés dont la technologie était très innovante, mais qui ne disposaient pas d'une capacité financière et d'une gestion leur permettant d'affronter la concurrence .

Le second «pic» de création (1998-1999-2000) doit probablement être attribué conjointement à la bonne santé du secteur et aux mesures actives d'aide à la création initiées par l'Inria. Les créations sont principalement liées aux nouveaux besoins en relation avec Internet. Au fil du temps, ces créations sont de moins en moins des «intermédiaires » avec les grands donneurs d'ordre et de plus en plus des futures PME ayant soit un accès direct au marché des consommateurs, soit un accès médiatisé par d'autres PME. Les disparitions de sociétés correspondent pour la majorité d'entre elles à des rachats ou des fusions. Dans d'autres cas, les technologies développées ont été reprises sous des formes diverses par d'autres entreprises. Les créateurs de ces sociétés «disparues » ont par la suite été à l'origine d'autres «jeunes pousses». Il faut toutefois remarquer que comme pour l'ensemble des sociétés technologiques, la vie des jeunes pousses issues de l'Inria est, dans leur première année, fort mouvementée.

Ces sociétés ont des créneaux d'activité très divers. Elles sont le plus souvent de type technologique mais depuis quelques années se créent aussi des sociétés de type marketing.

La «réussite» de ces différentes sociétés dans l'espace industriel est difficilement comparable. En effet, au delà de leurs places différentes dans le processus technologique, elles ont été créées à des périodes différentes, elles n'ont pas toutes bénéficié des outils d'aide Inria, et n'ont pas été confrontées au marché financier et au marché des produits dans des

\footnotetext{
${ }^{1}$ Les 26 essaimages effectifs au 31/12 1998 ont générées environ 1130 emplois et un chiffre d'affaires de 100 millions d'euros: Informations internes de l'Inria.
} 
circonstances analogues. Le choix des deux zones et des essaimages étudiés à l'intérieur de chacune des zones tente de prendre en compte cette variété (CF Annexe méthodologique).

Globalement, les essaimages des deux zones se distinguent par le type d'innovation qui les supporte dans la construction de leur projet, par leurs liens avec leur environnement et par la professionnalité de leurs acteurs.

- Les essaimages de Sophia se créent en spécifiant une connaissance générique en vue de satisfaire un besoin du marché ; ceux de Grenoble partent de l'identification d'un besoin et s'interrogent sur l'adaptation d'une technologie Inria à ce marché. Pour les premiers, la création de l'essaimage correspond à la poursuite logique et linéaire du processus d'invention commencé dans l'équipe de recherche. Le produit ou le processus proposé est nouveau. Pour les seconds, il s'agit le plus souvent d'un bricolage technologique qui, à partir des travaux de recherche des équipes de l'Inria et d'autres expériences professionnelles, permet de mettre au point ou d'améliorer un produit ou un service. Dans le premier cas, l'innovation est plutôt de type radical, alors que dans le second elle serait de type incrementale.

- Dans le premier cas, la concurrence est ouverte au marché international, dans le second plus limitée, l'innovation nécessite la proximité du client.

- Dans le montage du dossier de création, les créateurs de Sophia soignent l'aspect technologique, alors que ceux de Grenoble prennent du temps à définir les étapes de la construction de leur organisation et leurs besoins en ressources humaines. Les premiers privilégient la recherche d'investisseurs, les seconds celle de clients.

- Le lien de la société technologique de Sophia reste le plus souvent exclusif avec l'Inria, alors que celui de Grenoble est ouvert sur un environnement immédiat.

- Les créateurs sont plus jeunes à Sophia et n'ont que rarement une expérience professionnelle autre que celle de chercheur académique, tandis qu'à Grenoble, ils ont une professionnalité qui s'est construite à la fois dans la recherche académique et dans la recherche appliquée. De plus sur ce site, les créateurs ont une connaissance de l'entreprise et ont le plus souvent déjà participé à la création d'un essaimage.

- A Sophia Antipolis, le passage d'une entreprise fondée autour d'une personnalité à une PMI/PME est l'objet de tensions et de conflits, alors qu'à Grenoble, cette évolution est pensée et gérée depuis la création.

\section{II- Les déterminants de ces créations}

Ces déterminants sont liés à un effet institutionnel, à un effet territoire ou aux pratiques des acteurs locaux.

\section{II-1 Le dispositif institutionnel : La politique de transfert de l'Inria.}

L'Inria a été créé en 1967 par l'Etat en tant qu'organisme public de recherche appliquée: sa vocation a été, dès l'origine, de contribuer à l'essor du tissu industriel ${ }^{1}$. Sa mission est circonscrite au secteur de l'informatique et de l'automatique. Il dispose de moyens qui lui sont propres (unités de recherches, chercheurs, ingénieurs, techniciens et administratifs), mais il bénéficie aussi de liens forts avec l'université et le $\mathrm{CNRS}^{2}$. Sa

\footnotetext{
${ }^{1}$ Il est un des maillons du Plan Calcul.

${ }^{2}$ Ces centres de recherches accueillent aussi des chercheurs du CNRS et des universités.
} 
position et son rôle d' «institution » hybride entre la science et l'industrie sont alors originaux dans le contexte français de l'époque.

Depuis, les missions des institutions scientifiques se sont élargies à la valorisation des résultats de la recherche, ce qui a provoqué, dans le contexte actuel de diminution des crédits publics, un processus de coopération/compétition entre l'Inria, le Cnrs et les universités. Les tensions entre ces organismes portent sur les avancées scientifiques mais aussi sur la captation des contrats industriels et sur le nombre de création d'entreprise effectuée. La compétition qui était jusqu'alors limitée aux luttes de légitimité au sein de la communauté scientifique ou aux luttes de pouvoir au sein du système d'enseignement supérieur et de recherche s'est élargie. En effet, les collaborations industrielles ont une utilité directe dans le financement des recherches et donc dans la capacité à faire de la recherche. Elles sont par conséquent l'objet d'une concurrence entre équipes travaillant dans le même domaine. Le nombre d'entreprises créées devient un signe de la capacité des institutions et des unités de recherche à diffuser leurs savoirs et leurs savoir-faire dans le monde économique. Il permet ainsi de justifier les dotations budgétaires de l'Inria auprès des pouvoirs publics et de se maintenir en tant qu' « infrastructure institutionnelle ».

Pour garder sa spécificité et son rang dans la compétition entre institutions et donc ses crédits, l'Inria s'appuie, en particulier, sur des politiques et des outils facilitant la création d'entreprise. Sa direction s'est dotée (avant les autres organismes de recherche, les universités et les régions) de moyens lui permettant de supporter des acteurs «intermédiaires » entre la recherche et les entreprises, comme un système d'incubation, ou une politique de gestion de la propriété industrielle souple.

L'organisme utilise aussi au mieux certaines des facilités de gestion que lui concède son statut public pour aider à la création d'entreprise : contrats à durée déterminé et mise en disponibilité pour les titulaires de la fonction publique... Ainsi, les emplois d'ingénieursexperts sont prévus pour développer un produit ou un service issu de la recherche, avant que celui-ci ne soit approprié par un industriel. Des post-doc «création d'entreprise » sont réservés aux jeunes créateurs non-titulaires pendant la phase d'incubation de leur projet, tandis que les «senoirs » créateurs peuvent bénéficier de postes d'accueil.

L'Inria possède une filiale à $100 \%$, Inria Transfert chargé de l'incubation thématique des entreprises. Celle-ci apporte ${ }^{1}$ au créateur ses compétences (conception du plan de développement de l'entreprise, organisation de l'entreprise, support juridique, assistance administrative...), son réseau de relations et un local. Elle est fondatrice d'un fond d'amorçage privé auquel elle soumet des projets (ce qui leur donne une crédibilité scientifique). Toutefois, ce dernier garde la disponibilité de financer ou non l'essaimage.

Par ailleurs, l'organisme a choisi d'avoir une politique de propriété industrielle qui ne soit plus patrimoniale, mais qui facilite la création. Auparavant, il préconisait un remboursement «croisé », progressif compte tenu de l'évolution du chiffre d'affaires de l'entreprise et dégressif pour tenir compte de l'évolution du produit (le poids des connaissances transférées diminue dans le temps), ce qui conduisait à grever la trésorerie de l'entreprise au démarrage. Maintenant, il préfère négocier des redevances fixes, non dépendantes du chiffre d'affaires pendant les premières années (pendant 2 à 3 ans), en prévoyant une option d'achat des droits patrimoniaux à coût déterminé et avec Bons de Souscription d'action (BSA). Ce système permet au créateur d'avoir une stratégie flexible en fonction des premiers résultats de sa société et aussi d'apprendre son métier d'entrepreneur.

L'Inria est devenu, au fil du temps, une institution qui a acquis, au-delà d'un simple support de la politique nationale d'aide à la création d'entreprise, une réelle capacité institutionnelle à propulser des essaimages.

\footnotetext{
${ }^{1}$ Signature d'une convention d'incubation
} 


\section{II-2 Le rôle des territoires ou les deux espaces d'innovation localisés}

Les deux espaces d'innovation localisés pris en compte ne sont pas à priori comparables: l'un est une technopôle créée récemment par une décision des pouvoirs publics ; l'autre est délimité par l'influence d'une ville et par un territoire administré (un département). Toutefois, tous deux ont une forte activité dans le domaine des technologies de l'information et de la communication et possèdent une implantation d'une unité de recherche de l'Inria.

\section{II-2-1 La technopôle renversée.}

La technopôle de Sophia Antipolis est dite «technopôle renversée »(Longhi 1999), dans le sens où elle s'est développée dans un contexte particulier par rapport aux autres sites de ce type, en particulier par rapport à Oxford. Sophia Antipolis était, lors de la création du parc (1975), un «espace vacant », dans le sens où le territoire d'accueil était vierge de toute installation. Les conditions d'un apprentissage collectif permettant le développement d'un milieu innovateur localisé (Storper 1993) étaient alors loin d'être remplies : il n'y avait pas, dans l'environnement du site, des savoirs et savoir-faire industriels de base, ni de tradition de créations d'entreprise, ni de présence d'universités ou de centres de recherche; les moyens de transport au niveau local étaient peu développés. Malgré tout, la région bénéficiait d'une capacité d'attraction due à son climat et à son potentiel touristique.

L'acteur principal à l'origine de la politique de structuration d'une technopôle sur ce site est l'Etat (la Datar, les représentants de l'Etat dans la région...) qui avait comme objectif de diversifier le développement économique de la région. Sa politique a consisté à développer les infrastructures et à inciter, d'abord les multinationales américaines et les laboratoires de recherche publique (Unités de recherche de l'Ecole des mines et de l'Inria) puis des PME, à s'installer sur le territoire. Depuis l'origine, les pouvoirs publics ont agi pour que la technopôle soit le support privilégié du développement des filières liées aux technologies de l'information et de la communication. Cette spécialisation s'est étendue, par la suite au secteur de la santé (implantation de laboratoires Cnrs et Inserm).

Au cours des années 1990, L'Etat s'est quelque peu effacé, permettant ainsi l'émergence d'acteurs publics locaux diversifiés mais peu coordonnés entre eux. Ainsi, certaines «personnalités », médiatrices entre le monde politique et le monde économique, ont accompagné ce processus et ont apporté chacune leur propre contribution à la construction du dispositif actuel. Ces acteurs «publics » ont ainsi contribué, d'une part à orienter les actions de l'Etat, d'autre part à animer la technopôle de diverses façons, notamment en créant de multiples associations, supports de la création d'entreprise; ils ont aussi été à l'origine de l'émergence de conflits de pouvoir qui ont créé une opacité certaine pour les investisseurs et les entrepreneurs. Comme ils sont issus de l'élite économique et financière de la Côte d'Azur, ils ont contribué à valoriser l'esprit patrimonial de l'entrepreneur, à légitimer la force du capital financier et à insérer la technopôle dans la région.

L'université de Nice n'a accepté d'ouvrir une antenne à Sophia-Antipolis qu'en 1986 : elle a tardé à développer les différentes filières présentes aujourd'hui (Ecole d'ingénieurs dans le domaine des technologies de l'information et de la communication -TIC-, de commerce...), à déployer sa capacité de recherche et à s'insérer dans le milieu économique local. Comme les centres de recherches transférés de la Région parisienne ont continué de préférer les relations qu'ils avaient antérieurement avec les prestigieuses écoles et universités parisiennes, les interactions au sein de l'espace académique territorial sont encore réduites. A 
la fin des années 1990, le nombre des étudiants et chercheurs est limité ; les chercheurs des organismes publics ne sortent que rarement de leurs laboratoires ; les enseignants-chercheurs sont regroupés dans leurs propres unités de recherche...

Jusqu'en 1996, la croissance du territoire a été essentiellement exogène grâce à l'installation de multinationales américaines principalement dans le secteur des services ${ }^{1}$. Celles-ci ${ }^{2}$, dans le contexte de globalisation de l'époque, y voyaient une base d'implantation leur permettant d'adapter leurs produits aux marchés européens, puis des PME spécialisées dans le services aux grandes sociétés se sont créées. Depuis 1996, une dynamique de créations de «jeunes pousses » plus indépendantes des multinationales se développe. Toutefois, si ces créations s'effectuent dans le secteur des TIC, elles relèvent plus de sociétés commerciales (ouverture de portails sur Internet) que de sociétés technologiques.

Les cadres ou les techniciens supérieurs du secteur privé comme du secteur public sont les catégories sociales largement majoritaires de la zone où la densité de matière grise au $\mathrm{m} 2$ est importante. Il existe ainsi une certaine homogénéité de la population, ce qui paradoxalement rend les rapports sociaux peu conflictuels mais limite la dynamique relationnelle sur le territoire. Par ailleurs, le caractère récent et «renversé » de la technopôle, le coût élevé de l'habitat qui répartit les différents acteurs de la technopôle sur tout le département selon des critères de revenus limitent les relations sociales informelles entre les individus. Certains liens construits au cours de l'activité de travail se poursuivent dans la sphère domestique mais rarement. Les associations tentent de remédier à cela, en créant du lien grâce à une formalisation des rencontres. Mais elles privilégient avant tout les contacts entre acteurs économiques légitimes.

Globalement, la technopôle de Sophia-Antipolis est dans une situation paradoxale. Alors que les différents acteurs économiques de ce territoire apparaissent incompatibles entre eux comme l'attestent tant de conflits résultant de leur mise en concurrence (les associations, les institutions académiques etc.), cette incompatibilité a créé un climat d'émulation qui s'est peu à peu transformé en «milieu innovant »(Longhi 1999), au fur et à mesure qu'ils acquièrent une réelle capacité d'apprentissage collectif. Cette technopôle a donc engendré un certain type de «culture » qui permet de socialiser les acteurs économiques au contexte concurrentiel fort du secteur des hautes technologies. Elle conserve une image très valorisante comme symbole de la «nouvelle économie » et certaines entreprises déménagent pour profiter de cet avantage (Zimmerman 2000). Toutefois, ce milieu reste très opaque dans les systèmes d'incitations et d'aides qu'il propose aux créateurs. Les acteurs publics locaux tardent à construire leur propre cohérence et leur cohésion. Le capital matériel et symbolique de la technopôle reste fragile.

\section{II-2-2 Le système local d'innovation de la région grenobloise:}

Grenoble et le département de l'Isère constituent un «système local d'innovation" dans le sens proposé par Gilly et Grossetti (1993). L'ancienneté du développement industriel (Morsel, Parent 1991) et la force des accumulations en connaissances technologiques de base, la lente construction de routines de collaboration entre la formation supérieure et la recherche, les entreprises et les acteurs publics, ont facilité la circulation des savoirs et des savoir-faire, et ont favorisé la coopération entre acteurs et la dynamique technologique sur un même

\footnotetext{
${ }^{1}$ Pour créer un effet d'entraînement sur les grandes entreprises internationales, des entreprises nationales et souvent nationalisées sont incitées à délocaliser certaines de leurs filiales (Air France, Thomson Sintra, création de la Telecom Valley).

${ }^{2}$ Digital, Questel, Télémécanique, centre de réservation d’Air France, Dow Chemical.....
} 
territoire. La proximité organisationnelle a été un support de la proximité géographique (Rallet, Torre 2001)

Dès le début du $20^{\mathrm{e}}$ siècle, se crée autour de Grenoble une «symbiose » ou une «conjonction» (Bernardy de Sigoyer, Boisgontier 1988, Grossetti 1995) entre un milieu industriel local actif et des personnalités scientifiques qui permet à l'hydroélectricité de devenir le moteur du développement économique et scientifique. L'histoire de l'entreprise Merlin Gerin est ainsi un exemple parmi d'autres de cette construction conjointe de l'espace industriel, de l'espace de la R/D et de l'espace de formation supérieure. Elle met aussi en valeur le rôle de certaines personnalités dont la professionnalité s'est construite par des mobilités successives entre chacun de ses espaces.

Cette symbiose a marqué la construction de chacun des espaces et a favorisé l'institutionnalisation d'un espace intermédiaire entre eux. Elle s'est poursuivie après guerre et a spécifié le système industriel grenoblois comme l'attestent à la fois la spécialisation ancienne dans des technologies avancées, la croissance des contrats industriels avec le monde académique et l'émergence de sociétés technologiques sur la base de résultats de recherche.

Ainsi le tissu industriel est très varié et très interactif. La spécialisation de la zone autour des TIC s'est faite dans des ajustements permanents entre la micro-électronique, l'industrie de l'informatique et l'intégration de systèmes. A l'intérieur de ce secteur, l'informatique est largement supportée par la présence de deux grands constructeurs (Hewlett Packard et Bull) qui ont permis la création ou l'implantation de SSII de taille européenne comme Cap Gémini et Sema Group et de PME-PMI. La forte croissance des contrats industriels enregistrée (Pestre 1990) s'explique d'une part par l'implantation d'entreprises à fort potentiel technologique et de leurs laboratoires de R/D (exemple CII) et d'autre part par le développement de sous-traitance de haut niveau réalisée par des PME pour les grands donneurs d'ordre de l'industrie et de la recherche (les industriels mais aussi le CEA, les laboratoires de L'IPG...). Ce milieu a été à l'origine de la création de nombreux essaimages issues soit des entreprises industrielles, soit de l'espace académique ou d'une collaboration entre eux. De nombreuses histoires d'entreprises (Sames 1945, Télémécanique 1961, ST Microelectronics 1974....) confirment l'ancienneté de la capacité du territoire de Grenoble à créer des entreprises ou à transférer des produits de la recherche dans le monde économique.

Ce milieu est aussi à l'origine d'un des premiers pôles français de recherche et d'enseignement supérieur. Contrairement à la technopôle de Sophia, le couple formationrecherche en est le ferment. Les universités, les écoles d'ingénieurs et les centres de recherche (CNRS, CEA...) ont collaboré depuis l'origine. Ensemble, ils entretiennent des relations avec l'espace industriel et cohabitent de plus en plus souvent avec les entreprises sur des ZIRST (Meylan, Montbonnot etc.). Ce pôle est fortement orienté vers les technologies de l'information et de la communication ( $6 \%$ des emplois totaux. Cf annexe). Les institutions académiques ont une renommée dans ce secteur et participent activement aux créations d'entreprises (le $\mathrm{LETI}^{1} \mathrm{du} \mathrm{CENG}^{2}$, le $\mathrm{LAG}^{3}$, l'IMAG ${ }^{4} \ldots$...). Ainsi les essaimages créés majoritairement issus soit de la recherche, soit des grandes entreprises ${ }^{5}$ ont le plus souvent un contenu d'innovation technologique fort.

Contrairement à Sophia, l'Etat n'est que peu intervenu sur le territoire ces dernières années, les personnalités grenobloises industriels ou scientifiques se méfiant, à l'instar de

\footnotetext{
${ }^{1}$ Laboratoire d'Electronique, de Technologie de l'Information.

${ }^{2}$ Centre d'Etudes Nucléaires de Grenoble.

${ }^{3}$ Laboratoire d'Automatique de Grenoble.

${ }^{4}$ Institut d'Informatique et de Mathématiques Appliqués de Grenoble.

${ }^{5}$ Sur les 22 entreprises que ce secteur a crée ces deux dernières années, la grande majorité sont issues de grandes entreprises( 3 dont Hewlett-Packard, Cegetel), ou de la recherche publique (13 dont 3 de l'inria et 5 du LETICEA) une a pour origine un GIE INPG Radiall Schneider, 4 sont « indépendantes.
} 
Louis Neel (Pestre 1990), du centralisme parisien. Par contre, la présence des acteurs publics régionaux sur le système local d'innovation est très prégnante, que ce soient les politiques ou les collectivités locales. Ils n'agissent pas indépendamment les uns des autres comme à Sophia, mais de façon coordonnée avec les autres acteurs de la triple hélice. Ils veillent particulièrement à ce que les services déconcentrés de l'Etat (Anvar et Datar, Direction de la Recherche et de la Technologie) s'engagent sur les dossiers régionaux et prennent l'initiative de la création des ZIRST. Ces acteurs publics locaux ont, plus qu'à Sophia, une expérience professionnelle individuelle qui les lient à ce milieu grenoblois de la symbiose. Ils sont le produit de cet espace local d'innovation qui valorise la figure de «l'entrepreneur technicien » (Grossetti 1995), ingénieux, astucieux et travailleur et moteur de l'innovation. Ce milieu grenoblois interagit aussi avec un capitalisme familial industriel qui continuent de financer l'innovation locale et de soutenir l'entrepreneuriat comme il l'a fait de tout temps.

Ce milieu réunit une population active très diversifiée où l'ensemble des catégories sociales du pays est représenté: les salariés des grandes et des petites entreprises industrielles ou de service, les cadres et les diplômés de l'enseignement supérieur, les entrepreneurs et les commerçants. Dans ce système social, du fait de son ancienneté et de sa construction progressive, le système d'innovation et les acteurs qui y interagissent sont valorisés: «Le chercheur est ici quelqu'un de reconnu, pas seulement du maire de sa commune (il s'agit de Montbonnot), mais aussi de la boulangère ». Les acteurs individuels d'innovation ont entre eux, grâce à la proximité géographique, des relations sociales en dehors du travail qui augmentent leur capacité d'échange ( les relations de foot»). L'imbrication des relations informelles entre individus conforte la symbiose entre les acteurs collectifs. Ceci n'empêche pas les tensions et les conflits liés à cette symbiose. Ainsi au début des années 1970, les collaborations académie/industrie étaient décriées par certains chercheurs et enseignants. Actuellement, après de nombreux débats et controverses productives, elles se sont imposées et ont crée des routines organisationnelles de coopération entre les différents acteurs qui n'excluent cependant pas les tensions comme avec n'importe quel partenaire. Elles sont aussi l'objet indirect de la compétition dans le champ scientifique, car elles sont un moyen de la recherche.

Le système d'innovation autour de Grenoble s'est constitué dans le temps sur une relation de proximité (géographique et organisationnelle) entre les acteurs industriels, les acteurs publics et les acteurs de l'enseignement supérieur et de la recherche. Cette relation a permis que la figure de «l'entrepreneur technique » soit valorisée dans les relations de travail comme dans les relations sociales plus larges. La création de sociétés technologiques issues de la recherche publique ou de la recherche des grandes entreprises reflète cette tradition ancienne bâtie sur une pratique des contrats industriels devenue routinière. Ce milieu peut être analysé comme le résultat de la confiance entre les différents agents économiques (Bernardy et Boisgoutier 1988), ou plutôt le produit de la construction des acteurs dans un "même creuset » (Lanciano et ii 1998), ce qui aurait pour effet de faciliter un "alignement » (Callon 1998) des pratiques professionnelles les unes sur les autres. Comparée avec Sophia Antipolis, la cohérence de ce système est, d'une part, liée à la «routinisation » des pratiques de collaboration des acteurs plus qu'à l'action d'une politique publique. D'autre part, elle crée des irréversibilités plus fortes dans sa trajectoire: cette zone a une moindre flexibilité pour s'adapter aux changements de régimes technologiques que la technopôle (Granovetter1983 ).

\section{II-3 La construction des pratiques et des routines de recherche à l'Inria à Sophia Antipolis et à Grenoble.}


Les chercheurs de l'Inria ont en commun une conception de l'innovation qui est en relation avec leur organisation collective et leurs pratiques.

Leur expérience même les amène à penser que le processus d'innovation n'est pas linéaire. Il comprend des boucles de rétroaction entre du fondamental et de l'appliqué (Kline et Rosenberg 1986) qui les «obligent» à travailler avec les industriels. Il existerait ainsi une «boucle vertueuse » entre la recherche et l'industrie qui ferait que chacun aurait intérêt à travailler avec l'autre : ainsi, pour la recherche académique, l'industrie représenterait «le monde des usages »; elle serait un relais entre la recherche et la société ; elle fournirait des «questions», des «problèmes à résoudre » qui seraient autant d'incitations à la recherche fondamentale. Sûre de l'efficience de la relation science-industrie, la direction de l'organisme comme les chercheurs interrogés ne mésestiment pas pour autant les «carcans séparés »dans lesquels se trouvent les acteurs de l'innovation. Ils sont dans des temporalités qui les opposent : leur vision stratégique, le temps qu'il leur faut pour prendre une décision, leur durée d'implication dans le processus, la pérennité de leur intérêt sont différents. Ils n'ont pas, non plus, le même langage, ni les mêmes objets de recherche. Leurs modes d'incitation et d'évaluation s'opposent. Une division des tâches semblent s'imposer pour les acteurs de l'innovation interrogés: la recherche académique a comme premier objet de produire des connaissances génériques (qui peuvent être entre autres des algorithmes), alors que l'industriel veut pouvoir intégrer dans son propre processus de production un logiciel qui soit adapté à ses propres besoins. Mais entre ces deux fonctions, il reste plusieurs phases (faisabilité, prototypage, développement - rôle du code et de sa «robustesse » - et passage d'une production en petite quantité à une production en grande quantité) que chacun considère comme étant du domaine de l'autre.

Ce «gap » doit être repensé et comblé, pour que la relation puisse s'engager entre les deux partenaires. Pour ce faire, d'une part, l'Inria sélectionne, socialise et professionnalise les chercheurs et les ingénieurs de façon à faciliter leurs liens avec les entreprises. D'autre part, il s'est doté d'une organisation collective adaptée aux relations avec les différents acteurs du monde industriel (spécificité des PME par rapport aux grandes entreprises) et aux différentes situations rencontrées.

Cette politique est institutionnelle et nationale. Dans les deux zones observées, elle est cependant infléchie par les systèmes locaux d'innovation et par les conditions d'implantation de l'Inria sur les territoires.

L'implantation de l'Inria à Grenoble est tardive (1992) par rapport à la constitution de ce système d'innovation local et par rapport à son installation sur la Côte d'Azur (1982). Alors que l'Inria à Sophia est un des acteurs de la création de la technopôle à partir d'un espace vacant, à Grenoble, l'organisme public s'installe dans un système local d'innovation constitué. Alors que l'Inria Sophia s'est installé sans avoir construit préalablement de relations avec le système de formation supérieure - ce qui a retardé les liens formationrecherche-, l'implantation de l'Inria Grenoble a été conçue et réalisée pour être insérée dans le tissu universitaire.

La formation, les pratiques et les référents des chercheurs ne sont pas non plus identiques dans ces deux zones.

\section{II-3-1 La formation des chercheurs.}

La formation d'origine des chercheurs de l'Inria n'est pas reconnue comme déterminante pour leur carrière. Les doctorants sont ainsi issus de cursus scolaires en informatique très variés: grandes écoles d'ingénieurs, écoles d'ingénieurs rattachées aux universités, filières universitaires. Toutefois, un nombre notable de directeurs de recherche de l'Inria sort des «grands corps»(Polytechnique, Normale...). Il est difficile de savoir si ce 
phénomène est un effet de génération lié au rôle des «pionniers» de la «Science informatique » en France ou si le passage par les grandes écoles constitue un avantage comparatif réel, ce qui serait une information sur le type de sélection de l'Inria. Cette différenciation entre national et local se recoupe avec le choix d'un recrutement plus académique et général (les grandes écoles d'ingénieurs parisiennes) et un recrutement plus technique et professionnalisé (les écoles d'ingénieurs de province spécialisées dans les TIC).

Selon les territoires, les unités et les équipes de recherche de l'Inria privilégient différemment les étudiants issus des grandes écoles parisiennes ou des DEA ayant une renommée scientifique nationale ou les systèmes d'enseignement supérieur locaux. Ainsi, l'Inria Sophia, qui n'a pu recruter sur place faute d'offre au début de son installation, a conservé ses habitudes antérieures et recrute majoritairement au niveau national. Par contre, l'Inria Grenoble diversifie plus ses embauches en raison de sa connaissance des ressources humaines produites par le système local d'innovation. De la même façon, le recrutement de Sophia semble s'appuyer sur la valorisation de connaissances générales et académiques, tandis que celui de Grenoble est plus tourné vers des savoirs et savoir-faire spécialisés et professionnalisés.

Et c'est en combinant ce choix de recrutement et l'organisation de collectifs de travail souples et orientés vers des objectifs précis que les chercheurs permanents contribuent à socialiser et à former les jeunes générations.

\section{II-3-2 Les pratiques de recherche des équipes « projets ».}

L'Inria est organisé en quatre thèmes de recherche ${ }^{1}$ dans lesquels se retrouvent des projets, ce qui contribue à construire une ressource humaine spécifique. La politique de l'Inria n'est pas de spécialiser à priori les unités de recherche des différentes régions sur un thème. Et effectivement, les équipes de recherche des centres de Sophia et Rhône-Alpes se répartissent entre les quatre thèmes ${ }^{2}$.

Chaque projet est créé pour atteindre un objectif scientifique déterminé. Il réunit une équipe de 30 à 40 personnes (dont 10 à $15 \%$ de personnels permanents). Cette organisation, où les thésards et les chercheurs «post-doc » ont une place essentielle, crée un lien fort entre le chercheur encadrant/enseignant et le chercheur en apprentissage. Ce lien est à la base du travail collectif et de son évaluation ${ }^{3}$. Les effets de cette évaluation ont tendance à favoriser la mobilité thématique des chercheurs, à leur imposer une certaine flexibilité et à les garder dans un état d'éveil permanent vis-à-vis des nouvelles trajectoires scientifiques et technologiques.

Si cette organisation se retrouve dans l'ensemble des unités de recherche de l'Inria à travers la France, le socle territorial l'infléchit. Elle est, en effet, appropriée différemment par les équipes de recherche selon les territoires et la configuration de leurs différents acteurs ${ }^{4}$.

\footnotetext{
1 1-Réseaux et systèmes ; 2- Génie logiciel et calcul symbolique ; 3- Interaction homme-machine , image, données, connaissances ; 4- Simulation et optimisation des systèmes complexes.

${ }^{2}$ même si le thème 2 n'est que faiblement représenté (une équipe) en Rhône-Alpes.

${ }^{3}$ L'évolution des travaux de l'équipe est évaluée, tous les 4 ans, à partir des résultats scientifiques obtenus par rapport à cet objectif. Cette évaluation consiste en un échange d'avis, sous forme de séminaire de quelques jours, entre le responsable de l'équipe qui explicite et défend son rapport d'activité et des experts du domaine issus de la recherche académique (francophones ou non francophones) et ceux de la recherche industrielle. Cette évaluation s'avère stricte et effective, dans la mesure où le taux de renouvellement des projets (arrêt + démarrage) est élevé :

En 2001, sur 87 équipes-projets existant au $1^{\mathrm{er}}$ janvier, 11 ont été crées dans l'année, 13 ont été supprimés.

- A Sophia, sur 29, 6 projets ont été créés, 5 ont été arrêtés.

- En Rhône-Alpes, sur 18, 3 projets ont été créés, 3 ont été arrêtés.

${ }^{4}$ Ainsi, à Sophia Antipolis, les équipes de recherche sont majoritairement des unités propres à l'Inria (22 unités propres et 8 en partenariat) au contraire de Rhône Alpes ( 7 unités propres et 11 unités en partenariat). A
} 
L'insertion de l'Inria Rhône Alpes dans le système universitaire - en particulier ses liens avec les écoles d'ingénieurs, sa création récente et sa croissance rapide - justifie la présence comparativement beaucoup plus importante d'enseignants-chercheurs dans ses équipes (40\% de chercheurs statutaires de l'Inria dans les équipes de l'Inria en Rhône-Alpes et $85 \%$ à Sophia) et de chercheurs ayant des expériences professionnelles antérieures (dans les laboratoires de recherche publique ou d'entreprises). Cette situation explique en partie le poids des enseignements à l'Inria Rhône-Alpes par rapport à ceux effectués à l'Inria Sophia. La professionnalité différente des chercheurs des deux sites contribue à expliquer les formes des collectifs de travail (Amiot 1996).

L'organisation en projets se veut proche de celle des unités de R/D industrielle. Ce qui permet aux industriels d'avoir une bonne visibilité des résultats de la recherche, et donc d'insérer dans les programmes de recherche académique des orientations de recherche appliquée correspondant à leurs financements. Par ailleurs, cette organisation valorise aux yeux du chercheur l'importance des objectifs et légitime la volonté de mener une innovation jusqu'à sa mise sur le marché. Ainsi globalement, les équipes de recherches des deux unités de l'Inria sont notablement financées ${ }^{1}$ par les contrats industriels, même si on observe que la part des ressources propres varie énormément selon les projets. A Sophia, ces contrats sont majoritairement signés avec des grandes entreprises nationales ou internationales, en RhôneAlpes, les firmes sont en tendance de taille inférieure et plus régionales.

Ces différents éléments de comparaison justifient que les projets de recherche de l'Inria Rhône-Alpes soient plus ouverts sur le système local d'innovation et plus liés à la capacité du territoire à engendrer de l'entrepreneuriat technique, alors que ceux de Sophia sont marqués par l'excellence académique de l'institut national de recherche.

\section{II-3-3- Le référent des USA.}

Les USA sont souvent un référent incontournable dans les discours de nombreux chercheurs de l'Inria à la fois en terme d'excellence scientifique et en terme de pratiques d'innovation. La génération des chercheurs titulaires - "pionniers dans le contexte français »a été amenée à partir aux USA, pour s'initier aux nouvelles connaissances et technologies informatiques. Leurs formations initiales au sein du système éducatif français étaient très théoriques, mathématiques et formalisées par rapport à celles de leurs collègues américains. Ils ont appris ainsi, dans le milieu universitaire américain, à valoriser la recherche appliquée et l'activité en entreprises (les petits boulots et les stages d'été au cours de la thèse). De ces expériences, ces chercheurs ont, d'une part, construit leur problématique de recherche et la spécificité de l'Inria par rapport aux autres organismes de recherche (éloignement de l'académisme et autonomie par rapport aux critères d'excellence du système d'enseignement supérieur: thèse d'Etat), et d'autre part remis en cause l'incompatibilité entre recherche fondamentale et valorisation. La génération suivante n'a pas eu nécessairement à partir aux USA, pour acquérir des compétences qui étaient devenues disponibles en France. Mais la communauté de chercheurs à l'Inria continue, semble-t-il, à conserver le «patrimoine mental » qui remonte à cette première période: une attitude positive vis-à-vis du rapprochement science et technologie et surtout une approche cognitive en faveur de l'innovation. Les étudiants sont familiarisés par leurs aînés à cet «esprit » américain et surtout à son pragmatisme. En outre, ils profitent des réseaux de relations tissées par les anciens pour effectuer leurs «post-docs ». aux USA ou pour immerger dans le monde ultra spécialisé des essaimages ;

l'intérieur de ces partenariats, Sophia coopère au niveau national (2 partenariats), au niveau régional (2) et au niveau territorial (4), tandis que Rhône-Alpes ne possède que des unités mixtes régionales (3) ou territoriales (8). ${ }^{1}$ entre $20 \%$ du budget local pour Rhône-Alpes et $26 \%$ pour Sophia. 
Ce référent symbolique a été plus souvent cité, lors de nos entretiens à Sophia qu'à Grenoble, bien que l'on ne puisse distinguer les deux sites ni par les relations entretenues au sein de réseaux franco-américain, ni par la mobilité des seniors ou des juniors vers les USA.

La formation initiale, la construction de la professionnalité opérée dans l'organisation en projet et les divers référents des chercheurs s'avèrent être à l'origine, malgré l'aspect fondamental des recherches de l'Inria, de pratiques et de routines favorables au transfert technologique, mais différenciées selon les territoires. Les centres de recherches implantés sur les sites en région ont en commun ce référentiel (Jobert, Muller 1989) institutionnel, qui est différemment approprié selon le patrimoine (Godard, Salles 1991) territorial de chacun. Ainsi, les projets de recherche de l'Inria comme la professionnalité des acteurs de l'innovation de cet organisme sont des «construits » de l'institution nationale Inria, marqué par son histoire dans l'espace d'innovation français et de la dynamique propre aux territoires.

\section{Le rôle des effets institutionnels, des effets de territoire, de la construction des acteurs sur la création des essaimages.}

Selon les diverses positions théoriques, les essaimages peuvent être considérés comme des produits fabriqués par l'action publique ${ }^{1}$ pour faire l'intermédiation entre la recherche publique et le monde industriel. Ils peuvent émaner strictement des jeux d'acteurs décentralisés, en profitant du patrimoine, de l'opportunité et de l'interaction entre les acteurs au niveau territorial. Ils peuvent aussi être conçus comme des acteurs certes ayant des stratégies autonomes fondées sur leurs propres ressources et compétences, mais en même temps coproduits par un espace intermédiaire territorialisé entre l'espace de l'enseignement supérieur et de la recherche, l'espace industriel et l'espace public. Notre analyse tiendra compte de ces différentes interprétations, tout en privilégiant ce dernier positionnement.

\section{III-1 Les essaimages en tant qu'outil d'intermédiation géré par l'acteur public ou la suprématie de l'effet institutionnel}

Une partie des essaimages étudiés a été crée en raison principalement de l'absence d'industriels intéressés par le résultat scientifique d'une équipe Inria et capables de le mener jusqu'au développement technologique de façon à mettre sur un marché un nouveau produit ou un nouveau service. Cette absence de motivation industrielle est liée soit à la circulation insuffisante de l'information, soit à un défaut cognitif de la part de grosses entreprises trop bureaucratisées pour prendre des risques par exemple, soit à l'incapacité des chercheurs de l'Inria à convaincre des acteurs industriels de l'intérêt économique de leur «invention ». L'Inria en tant qu'institution représentant l'Etat soutient la création de sociétés technologiques

but de contribuer au développement économique national.

dans le

Dans ce sens, les essaimages jouent le rôle d'outils «intermédiaires » (Carlsson 1994, Bessant 1994, Dogson et Bessant 1996) qui remplacent les chaînons manquants (l'industriel) dans le processus d'innovation. Ils ont également un rôle de médiation entre les agents économiques qui interviennent dans les différentes étapes du cycle de production. L'institution publique contribue à combler ce vide, en «élargissant» ses frontières et ses fonctions au développement des produits et en proposant des outils de valorisation. L'espace d'innovation à l'origine des essaimages s'organise essentiellement autour de cette institution au niveau national comme au niveau territorial.

\footnotetext{
${ }^{1}$ Injonction de l'Etat à l'institution publique de faire preuve de son utilité sociale.
} 
L'Inria Sophia a été un élément de la stratégie de l'Etat dans la création de la technopôle : il a participé à la production de la légitimité scientifique et technologique de la zone; il a permis ainsi d'attirer des entreprises, des institutions universitaires et des investisseurs. Mais tout compte fait, son intégration dans le réseau local reste faible. Les outils d'incubation proposés par l'Inria sont incontournables pour les chercheurs-créateurs de Sophia. Leur isolement sur la technopôle les conduit à des transferts directs entre l'équipe projet Inria et la société technologique, sans qu'il y ait intervention d'autres acteurs locaux. Ils privilégient la valorisation de résultats de recherche brillants à partir de leurs connaissances technologiques au niveau national et international. Les liens entre l'équipe de recherche et les créateurs se sont construits lors du travail scientifique et se prolongent pendant le développement qui est effectué de façon le plus souvent conjointe. Ils sont d'autant plus difficiles à rompre lorsque l'essaimage doit devenir une entreprise. Les créateurs proposent de combler le "gap» entre la recherche académique et le marché à partir d'une invention générique. Par contre, les relations entre les essaimages Inria sont faibles. Le réseau Inria de Sophia est essentiel pour les essaimages, mais il est très nettement centralisé autour du référentiel public Inria.

Si les essaimages de Sophia peuvent être analysés comme des outils d'intermédiation entre la recherche académique et l'industrie gérés par l'action public, il n'en est pas de même pour ceux de Grenoble.

L'Inria Grenoble s'est installé tardivement dans un système où les relations espace industriel/espace de l'enseignement supérieur et de la recherche/espace public sont à l'origine même du développement économique et scientifique. Il s'est habilement inséré dans ce milieu, mais il n'est qu'un des acteurs de l'innovation parmi d'autres. Les outils de transfert proposés par l'Inria ont contribué à réactiver la capacité du milieu grenoblois à créer des entreprises, mais ils sont concurrencés par d'autres outils proposés par des universités ou des associations entre acteurs publics et privés. Les créateurs de l'Inria Grenoble ont donc souvent eu le choix entre plusieurs types d'incubateur ou entre les services proposés. De même, leur insertion dans ce système local leur permet de multiplier les contacts avec d'autres unités de recherche académiques mais aussi avec d'autres industriels. Le processus d'innovation proposé est alors moins linéaire que dans le cas de Sophia Antipolis : il prend appui à la fois sur une bonne information sur le marché et sur la technologie. Les connaissances produites par l'équipe projet de l'Inria sont, dès l'origine, métissées avec d'autres savoirs et savoir-faire. Moins direct aussi, le processus de création de l'essaimage fait intervenir de multiples acteurs. L'origine Inria de celle-ci est moins exclusive : les essaimages sont à l'origine de la multiplication des relations entre eux et s'insèrent dans les réseaux d'autres institutions ou associations. Ses réseaux sont plus diffus et moins centralisés autour de l'acteur public que ceux de Sophia.

L'espace d'innovation construit autour d'une institution semble pouvoir expliquer le cas de Sophia, mais plus difficilement celui de Grenoble.

\section{III-2 Les essaimages comme produits des systèmes d'innovation locaux ou l'effet territoire.}

Les travaux relatif au «Systèmes Localisés d'Innovation » (Gilly et Grossetti 1993, Grossetti 1994, Colletis, Gilly et Pecqueur 2001) cherchent à appréhender les systèmes d'innovation à un niveau méso, en élargissant la notion de méso-système productif (MSP) aux situations d'innovation (De Bandt 1991, Gilly 1987, 1992). La notion de MSP met en valeur la force de la proximité organisationnelle (Rallet 1991, Rallet et Torre 2001) dans la 
constitution des systèmes. Elle prend en considération aussi bien la dimension historique, les politiques publiques, les processus endogènes au niveau technico-productif et institutionnel, les processus exogènes liés à l'environnement. Un méso-système aurait ainsi une capacité collective endogène supérieure à l'addition des stratégies individuelles: l'intensité des relations de coopération entre entités institutionnelles marquerait sa qualité ; il aurait aussi un mode spécifique de gestion des ressources humaines ainsi qu'un type d'endogénéïsation des ressources et des contraintes extérieures particulières ${ }^{1}$.

Le système local d'innovation à Grenoble représente l'archétype du système d'innovation localisé par rapport à celui de Sophia. Il s'est constitué sur du long terme en une organisation efficace: les différents acteurs de la relation trilatérale ont des routines organisationnelles qui donnent de la cohérence à cet espace. L'adhésion à un même système de représentation et de règles facilite la coordination entre acteurs. Le système d'innovation grenoblois est un facteur déterminant de la création des essaimages. En revanche, la technopôle de Sophia Antipolis est récente : elle a été construite à partir d'une politique volontariste de l'Etat et des acteurs publics locaux ; la croissance des activités y paraît plus importante que leur complémentarité.

Ces histoires différentes expliquent la réactivité des deux zones aux changements conjoncturels. Le système grenoblois est plus robuste, il résiste à l'aléa brutal qu'est la chute de la bourse, sait s'adapter aux nouvelles contraintes du marché ou aux évolutions technologiques. Mais il a du mal à opérer des modifications substantielles de trajectoire sectorielle (dépendance de sentier). Le tissu économique de la technopôle de Sophia est plus fragile car plus dépendant de la conjoncture économique mondiale. Par contre, il a déjà fait preuve d'une capacité de flexibilité et de motricité importante, lors de «crises » successives.

D'après Rallet (1993), l'importance de la proximité géographique dans les processus d'innovation technologique doit être relativisée en fonction du degré d'intensité des interactions entre les agents et de l'importance des connaissances tacites. Or, la technopôle de Sophia Antipolis n'est que peu productrice de relations informelles/formelles entre acteurs (individuels ou collectifs) et de connaissances tacites, au contraire de Grenoble.

Les acteurs grenoblois de la création d'entreprise interagissent dans un milieu cognitif où sont valorisés l'entrepreneuriat et la culture technique autour de la micro-électronique ou d'une complémentarité entre les activités de constructeur informatique, d'éditeur de logiciels et d'intégrateurs. La figure du créateur est celle de l'entrepreneur-technicien, figure légitimée par la société au niveau local. Les créateurs de Sophia sont nés sous le signe d'Internet, de l'édition de logiciels. Ils sont liés à la figure emblématique du chercheur créateur de jeune pousse international. Pour les essaimages de Sophia, l'institution Inria a pour rôle de réduire les risques pris par le créateur, tandis qu'à Grenoble, c'est le système local d'innovation qui tient ce rôle.

\footnotetext{
${ }^{1}$ Gilly et Grossetti cherchent à articuler ces dynamiques industrielles qui renvoient à des stratégies spécifiques des organisations avec les dynamiques territoriales qui renvoient aux stratégies d'intégration de ces organisations au sein d'une zone socio-économique définie. Ils promeuvent ainsi les systèmes locaux d'innovation et la notion de proximité territoriale qui font interagir la proximité organisationnelle à une proximité géographique. L'inscription dans un même territoire favoriserait les relations entre acteurs sociaux (organisations et individus) en particulier grâce à leurs liens informels (utilisations des relations informelles par les organisations, Kreiner, Schultz 1993). Elle permettrait la création d'un contexte socioculturel favorable au croisement des compétences et des technologies, fondé sur des valeurs de réciprocité et de confiance entre acteurs (Von Hippel 1989). Colletis, Gilly et Pecqueur (2001) complètent cette approche en insistant sur un principe de cohérence sur lequel ces auteurs avaient peu travaillé et sur la proximité institutionnelle («adhésion des acteurs à un espace commun de représentation, de repères d'action et de modèles de pensée qui oriente les comportements collectifs »).
} 
La notion de système d'innovation localisé explique de façon suffisamment convainquante les créations grenobloises, alors qu'elle est moins explicative de celles de Sophia.

III-3 Les essaimages, acteurs de l'espace intermédiaire entre l'espace industriel, l'espace de l'enseignement supérieur et de recherche et l'espace public.

L'étude des relations trilatérales met en lumière l'importance des acteurs et de leur construction dans les processus d'innovation. Les acteurs liés à l'entreprise ne sont plus les seuls acteurs de l'innovation comme à la période précédente. D'autres catégories d'acteurs sont présentes dans le processus, tels que les universitaires et les chercheurs académiques, certains acteurs publics mais aussi des acteurs-relais et des acteurs hybrides entre les différents espaces de la triple hélice. L'interaction entre ces acteurs et ces espaces délimite un nouvel espace d'innovation que nous nommons « intermédiaire ».

Cet espace est structuré par une identification précise du rôle des acteurs du processus d'innovation, par l'organisation des pratiques de recherche et de transfert et par la façon dont se construit la professionnalité de l'ensemble des acteurs de l'innovation.

Il peut être appréhendé au niveau national. Il peut être aussi analysé comme un mésosystème au niveau d'un territoire, d'un secteur d'activité ou autour d'une institution et au niveau des pratiques locales de travail des acteurs. Ces différents niveaux interagissent entre eux, l'espace national contribue à influencer les pratiques territoriales et locales, comme les acteurs locaux participent de la constitution de l'espace territorial et national.

Les essaimages sont à la fois des relais actionnés par des personnalités ou par différentes catégories d'acteurs hybrides. Ils sont relais, car ils ont pour fonction de combler un «gap » entre le système de recherche et les entreprises. Ils sont portés par des acteurs dont la professionnalité a été acquise ou est en train de se construire à travers différentes expériences (mobilités) entre l'espace académique et l'espace industriel. La relation chercheur-créateur-manager est ainsi un facteur déterminant de la création d'entreprise technologique.

- Le chercheur-inventeur a, à la fois, la capacité «à inventer» une ressource générique et à imaginer sa spécification sur un marché. Le système d'enseignement supérieur est responsable de la formation initiale et des connaissances qui lui ont permis d'être sélectionné par l'Inria, organisme de recherche académique. L'équipe de recherche de l'Inria, organisme ayant une histoire particulière dans le système de recherche français, lui permet d'acquérir les savoirs et les savoir-faire professionnels du chercheur académique en informatique et automatique. L'équipe de recherche, résultat de l'interaction entre les stratégies de l'institution et les pratiques du territoire, l'aide à se construire une compétence de chercheur appliqué. Ce chercheur particulier est le produit des pratiques nationales et locales, c'est-à-dire de sa proximité géographique, institutionnelle et organisationnelle avec d'autres acteurs (entreprises, universitaires...), mais aussi de sa proximité cognitive avec ces mêmes acteurs, proximité qui lui permet des apprentissages communs ou complémentaires. Cette proximité cognitive est liée à l'acquisition de la professionnalité. Elle est construite dans le système de formation et autour des acquisitions communes ou complémentaires des expériences au cours de la vie professionnelle. Elle est créatrice de compatibilité entre les savoirs et les acteurs. Dans ce contexte, le chercheur a le choix de décider s'il devient ou non créateur de société technologique. Mais il reste de toute façon liée à elle en tant qu'inventeur.

- Le rôle du créateur est décisif (Mustar 1995, Flésia 1996). Toutefois, il peut être assez loin de la figure schumpetérienne classique qui renvoie uniquement à «l'entrepreneur individuel», puisqu'il est, au début du processus, soit inséré dans l'équipe de recherche académique, soit lié à cette équipe. Sa première tâche est de négocier avec ses collègues 
l'éventuel transfert de technologie du public au privé. Puis il définit l'objet économique de sa société, travaille sur l'application de l'invention, construit une stratégie d'organisation et de ressources humaines, cherche des investisseurs...A Sophia, le créateur est un chercheur de l'Inria ou plus souvent un «thésard ». Il est un des inventeurs du produit ou du service qui a choisi, souvent parce qu'il n'a pas accès à une carrière académique, d'utiliser pour son propre profit les mesures de transfert de l'institut. Il est jeune, lorsqu'il tente la création et n'a pas d'autre expérience professionnelle que celle qu'il a acquis à l'Inria comme apprentichercheur. Audacieux, il valorise à l'extrême le mythe du créateur schumpetérien. Il privilégie les conditions du transfert technologique, la période de démarrage et les relations avec les financiers. A Grenoble, le créateur est une figure plus diversifiée et plus âgée. Dans trois cas sur quatre, qu'il soit chercheur ou enseignant, il a aussi une expérience antérieure de la grande entreprise ou de l'essaimage, comme créateur ${ }^{1}$ ou comme salarié. Il n'est pas systématiquement inventeur du produit ou service mis sur le marché. Mais il construit son projet méticuleusement, sans précipitation, et il soigne, dès la période d'incubation, l'organisation et la gestion future de son entreprise. Il se préoccupe d'intéresser ses nouveaux clients et des marchés qu'il pourrait toucher.

Paradoxalement, la transformation du chercheur en créateur est moins courante à Grenoble qu'à Sophia. En effet, le développement du produit/service réalisé lors de la période de création correspond à la suite du travail de l'équipe de recherche : elle est souvent la dernière tâche collective effectuée par l'équipe-projet, entre ceux qui poursuivent leur carrière à l'Inria et ceux qui prennent le risque de partir². L'équipe Inria apprend alors un métier différent, celui de développeur, ce qui ne manque pas de modifier la professionnalité de chacun de ces membres. Les uns ne font qu'intégrer à leurs connaissances l'idée d'une finalité économique de la recherche, tandis que les seconds découvrent la nature et les contours de la $\mathrm{R} / \mathrm{D}$ industrielle, ce qui constituera leurs nouvelles compétences. Cette «fertilisation croisée » (Hatchuel, Sardas 1992) comme la réussite de cette expérience commune déterminent la qualité des relations entre l'équipe Inria et l'essaimage et surtout la poursuite ou non de leur collaboration. Elle contribue à l'espace intermédiaire au niveau local. Or, la construction de la professionnalité des chercheurs, à Grenoble, se fait au sein de la relation trilatérale dans une communauté large mais locale qui intervient naturellement dans le binôme inventeur-créateur. A Sophia Antipolis, la construction de la professionnalté des chercheurs s'effectue au sein de l'Inria et à partir des réseaux de l'organisme. A Grenoble, les chercheurs de l'équipe Inria restent peu impliqués dans les essaimages qu'ils ont contribués à créer, tandis qu'à Sophia, leur présence reste très prégnante dans le dispositif.

- Plus la création est lointaine, plus le rôle du créateur s'estompe ou disparaît. Ainsi au début du processus, le rôle du chercheur/développeur prédomine, puis c'est celui du créateur, ensuite lorsque l'essaimage devient entreprise, celui du manager (organisateur mais surtout «patron») devient premier. Les qualités requises pour remplir ces divers rôles sont différentes : ils peuvent être tenus soit par un même individu dont les compétences se «transforment » au fur et à mesure, soit par plusieurs personnes.

Le premier cas est le plus risqué puisque le chercheur doit pouvoir évoluer en créateur, puis en manager. En passant d'une fonction à une autre, il est amené, tout en profitant de ses expériences passées, à acquérir des compétences totalement nouvelles dans des champs disciplinaires éloignés de ceux de sa socialisation première (finances, droit, commerce,

\footnotetext{
${ }^{1}$ Dans un cas observé, le créateur a déjà déposé le bilan d'un essaimage: la direction de Grenoble lui fait confiance considérant «à l'américaine » que cette expérience ne peut qu'augmenter sa capacité à réussir la seconde fois, alors que celle de Sophia aurait plus tendance à considérer cela comme un échec.

${ }^{2}$ Le risque n'est pas le même que l'on ait une expérience professionnelle variée ou seulement une expérience de la recherche que l'on soit chercheur titulaire ou thésard.
} 
organisation..) et à s'écarter de certains de ses comportements antérieurs (processus de déapprentissage). Ce processus de transformation marque profondément la figure de cet acteur, l'essaimage et l'espace intermédiaire.

Dans le second cas, un nouvel acteur entre dans l'organisation avec des compétences reconnues, le plus souvent nommé par les actionnaires pour la fonction de manager. La relation inventeur-créateur doit s'ouvrir sur un nouvel acteur, le manager, ce qui se fait rarement sans tensions ou conflits. La relation inventeur-créateur-manager est délicate. Elle peut être supportée par des liens techniques ou professionnels, construits lors de la socialisation et de la professionnalisation de ces différents acteurs. Mais comme il est souvent difficile au créateur de retrouver un rôle dans l'entreprise, elle peut aussi entraîner le départ du créateur. Ce départ peut être à l'origine de la rupture entre l'inventeur et le manager, entre l'unité de recherche et l'essaimage et donc avec la source de nouvelles innovations technologiques.

Les créateurs de Grenoble ont déjà effectué la trajectoire de transformation de chercheur à créateur, ils ont souvent une expérience de manager. Ils se spécialisent dans le rôle d'«entrepreneur technicien » ce qui leur permet d'engager de jeunes chercheurs à la pointe du progrès et de rester le «patron »d'une entreprise où interagissent des savoirs et des savoir-faire différents. Ceux de Sophia ont été mieux évalués à l'origine par leurs partenaires financiers mais ils semblent plus subir la transformation de l'essaimage en entreprise que la diriger. Pour limiter le risque, ils acceptent qu'un gestionnaire extérieur leur soit adjoint. On observe que le processus de transformation de l'essaimage en entreprise va souvent de pair avec la prise de pouvoir du gestionnaire qui devient manager et le départ du créateur.

L'analyse qui consiste à mettre la relation inventeur-créateur-manager au centre des déterminants de la création de sociétés technologiques n'est pas contradictoire avec la prise en compte de l'effet institutionnel comme de l'effet de territoire. En effet, la construction des acteurs et la dialectique acteurs/espaces intègrent à la fois le niveau institutionnel national (l'Inria dans le système de l'enseignement supérieur et de recherche français, l'Inria comme vecteur des politiques de l'Etat), le niveau du territoire (le système local d'innovation ou l'espace intermédiaire au niveau local) dans les pratiques des acteurs.

\section{Conclusion}

Cet article tente de montrer la prégnance des acteurs dans la configuration des systèmes locaux d'innovation. Les essaimages en tant qu'acteurs intermédiaires entre l'espace d'enseignement supérieur et de recherche, l'espace industriel et l'espace public sont ainsi à la fois porteurs et résultats des logiques hétérogènes. Ils représentent ainsi un analyseur extrêmement intéressant, pour décomposer les effets multiples de l'interaction entre les différents espaces ou acteurs. Son cadre analytique fondé sur la notion «d'espace intermédiaire d'innovation » et sur la dialectique acteur/espace est à même de fournir des outils permettant de mieux contextualiser les logiques d'action et les jeux d'acteurs autour de la création des essaimages.

Autrement dit, l'analyse comparée des essaimages, proposée dans cet article, permet à la fois de déconstruire des formes de cohérence uniquement institutionnelle ou localisée, pour mieux saisir l'encastrement des logiques territoriales (région) et locales (site) dans une «matrice » nationale - infrastructure générique.

L'un de ses résultats les plus intéressants renvoie donc, par delà la diversité des pratiques et des logiques d'actions observées autour de la création des essaimages, à une certaine forme de cohérence entre les pratiques de divers acteurs selon la localité géographique. En effet, la politique conçue au niveau national en matière d'aides à la création 
des essaimages est différemment saisie et appropriée par les acteurs selon les constructions des espaces locaux d'innovation. Cette appropriation différenciée des outils reflète l'inscription de chaque site de l'Inria à la fois dans un milieu local ayant une certaine cohérence bâtie sur un ensemble des routines techniques, industrielles ou organisationnelles et dans une trajectoire historique différente de «milieu innovateur». Tout se passe alors comme si les acteurs encastrés dans chaque contexte local étaient fortement conditionnés pour créer leurs essaimages par le système cognitif au sein duquel ils se socialisent ainsi que par des ressources qu'ils trouvent à leur environnement proche.

Notre étude qualitative a toutefois une capacité limitée de généralisation des faits analysés, d'autant plus que les essaimages observés évoluent rapidement. Néanmoins, si l'interprétation que nous donnons à un ensemble de faits ouvre correctement une ligne d'intelligibilité, cela nous autorise, semble-t-il, une certaine lecture prospective sur les trajectoires futures de ces essaimages. Dans ce cas d'école, les essaimages de Sophia apparaissent mieux armés pour réussir leur mise sur le marché, parce qu'ils privilégient la relation inventeur-créateur et s'appuient sur la proximité institutionnelle avec l'Inria, réductrice d'incertitude initiale. Mais ils courent plus de risque dans leur transformation en entreprise. Par contre, les essaimages de Grenoble restent plus lents à se confronter au marché. Ils «travaillent» leur relation inventeur-créateur-manager, la maturation de leurs projets technico-industriels et leur encastrement dans les réseaux locaux. Ils ont sûrement plus de chance de survie et de capacité de durer en tant qu'entreprise. La poursuite longitudinale de ces essaimages nous permettra seule d'infirmer ou confirmer cette tentative de prédictibilité, difficile à assumer par les chercheurs en sciences sociales.

\section{Bibliographie}

AMIOT M, (1996), «Le laboratoire entre équipes et réseaux. Poids des techniques et conflits de légitimité ». Archives européennes de Sociologie. XXXVII.

AYDALOT P, KEEBLE D, 1988, «High-technolgy industry and innovative environnements in Europe : an overview », in AYDALOT P, KEEBLE D (Eds) High-technolgy industry and innovative environnements : The European Experience, Routledge, London.

BANDT J, 1991, «L'économie industrielle dans le contexte français : développement et spécificités », Traité d'Economie Industrielle . Economia.

BELLET M, COLLETIS G, LUNG Y, 1993 , «Economie et proximités », Introduction au numéro spécial de la Revue d'Economie Régionale et Urbaine, ${ }^{\circ} 3$.

BERNADY DE SIGOYER M, BOISGONTIER P, 1988, Grains de technopôle. Micor-entreprises grenobloises et nouveaux espaces productifs. Presses Universitaires de Grenoble.

BESSANT J, 1994, «Innovation and Manufacturing Strategy », in DODGSON, ROTHWELL(Eds), The Handbook of Industriel Innovation. Aldershot, Hants, Edward Elgar.

CALLON M, LAW J, 1995, “L'irruption des non-humains dans les sciences humaines : quelques leçons tirées de la sociologie des sciences et techniques ", in Les limites de la rationalité Reynaud, Bénédicte (dir.), Paris, La Découverte, 1996, vol. 2. Les figures du collectif, pp 99-118. 
CALLON M ,1998, «Introduction: The embeddnedness of economic markets in economics »in CALLON (Ed) The laws of the markets. Oxford Blackwell Publishers, Sociological Review pp1-57.

CARLSSON B, 1994, «Technical Systems ans Economic Performance » DODGSON, ROTHWELL(Eds), The Handbook of Industriel Innovation. Aldershot, Hants, Edward Elgar.

COHENDET P, 1996, "Recherche industrielle et processus d'innovation: le rôle structurant des pratiques de recherche ». Numéro Spécial "Recherche scientifique, innovation technique et politiques publiques". Sociologie du travail.

COLLETIS G, GILLY JP, PECQUEUR B, 2001, «Inscription spatiale des firmes, gouvernance des territoires et régulation d'ensemble ». Journées de la proximité, 13 et 14 décembre 2001 Paris.

COURLET C, PECQUEUR B, 1992, «Les systèmes industriels localisés en France : un nouveau modèle de développement » in BENKO, LIEPIETZ, Les régions qui gagnent. Collection Economie en liberté PUF.

DODGSON, BESSANT, 1996, Effective Innovation Policy : a new approach ». London International Thomson Business Press.

EDQUIST CH, 1997, Systems of Innovation, Technologies, Institutionss and Orrgaanisations, Pinter, London, Washington.

EZKOVITZ H, LEYDESDORF, 2000, «The dynamics of innovation : From National Systems and «Mode 2 » to a triple Helix of University-Industry-Governement relations ». Research Policy $n^{\circ} 29$ (a)

EZKOVITZ H, WEBSTER A, GEBHARDT C, TERRA BRC, 2000, «The Future of the university and university of the future : Evolution of ivory tower to entrepreneurial paradigm ». Research Policy $n^{\circ} 29(\mathrm{~b})$

FLESIA E, 1996, «La création d'entreprises par des chercheurs ». Notes de recherche-1432 CNRSLEST, Aix en Provence..

GIBBONS M, LIMOGES C, NOWOTNY H, SCWARTZMAN S, SCOTT P, TROW M, 1994, The New Production of Knowledge. Sage Publications.

GILLY JP,1987, Innovation et territoires : pour une approche méso-économique des technopoles, Revue d'Economie Régionale et Urbaine, $\mathrm{n}^{\circ} 5$.

GILLY, JP, 1992, Groups and new prospectives spaces : the case of Matra-Espace at Toulouse, in Spatial prospectives on regulation and competition, London, Dunford M. and Kafkala G. Eds.

GILLY JP, GROSSETTI M, 1993, Organisation, individus et territoires, le cas des sytèmes locaux d'innovation, Revue d'Economie Régionale et Urbaine, n³, p.449-468.

GODARD O, SALLES J-M, 1991, «Entre nature et société. Les jeux de l'irréversibilité dans la construction économique et sociale du champ de l'environnement» in BOYER, CHAVANCE, GODARD, Les figures de l'irréversibilité en économie. Les Editions de l'Ecole des Hautes Etudes en Sciences Sociales.

GRANOVETTER M, 1983, «The strength of weak ties », American Journal of Sociology, 87(1): 1360-1380. 
GRANOVETTER M, 1985, «Economic action and social structure: the problem of embeddedness », American Journal of Sociology, 91(3): 481-510

GROSSETTI M, 1995, Science, Industrie et territoire, Presses universitaires du Mirail.

HATCHUEL A, SARDAS JP 1992: "Le pilotage des systèmes complexes de production. Planification hiérarchisée et coopération dans l'incertain" in Tersac (de), Dubois eds, Les nouvelles rationalisations de la production. Cepadues.

HOTWAK MJ, GRAANTHAM CH-E, 2000, «The virtual incubator : managing human capital in the software industry ». Research Policy 29.

JOBERT B,MULLER P 1989, L'Etat en action. Politiques publiques et corporatismes. Paris, PUF.

KLINE ST, ROSENBERG N, 1986, An overview of innovation. In The positive sum strategy, Ed by LandayR and Rosenberg N, Academy of Engineering Press.

KRAINER K. and SCHULTZ M, 1993, «Informal collaboration in R\&D. The formation of networks across organizations », Organization Studies, 14(2): 189-209.

LANCIANO C., MAURICE M., NOHARA H., SILVESTRE J.J Eds, 1998, Les acteurs de l'innovation. Ed L'Harmattan

LONGHI C, 1999, « Networks, Collective Learning and Technology Development in Innovative High Technology Regions : The case of Sophia Antipolis. » Regional Studies, vol 33.4, pp 333, 342.

MAURICE M, SELLIER F, SILVESTRE JJ, 1982, Politiques d'education et d'organisation industrielle en France et en Allemagne. Essai d'analyse sociétale. PUF. Paris.

MASON, G. and WAGNER K. ,1999, « Knowledge transfer and innovation in Britain and Germany: "Intermediate institution" models of knowledge transfer under strain? », Industry and Innovation, 6 (1), 85-109.

MORSEL H, PARENT JF, 1991, Les industries de la région grenobloises. Presses Universitaires de Grenoble.

MUSTAR Ph, 1995, Science et innovation, annuaire raisonné de la création d'entreprises technologiques par les chercheurs en France. Economica. Paris

NELSON 1988, Institutions supportingtechnicalchange in US in Dosi G et ii Technical Change and Economic Theory. Pinter Publishers

NELSON (Eds), 1993, National Systems of Innovation: a Comparative Study » Oxford University Press.

PESTRE D, 1990, «Louis Neel, le magnétisme et Grenoble. Récit de la création d'un empire physicien dans la province française 1940-1965 », Cahier pour l'histoire du CNRS, n8. Editions du CNRS.

RALLET A, 1991, « Théorie de la polarisation et technopôle » Economies et sociétés $n^{\circ} 8$.

RALLET A, 1993, "Choix de proximité et processus d'innovation technologique », Revue d'Economie Régionale et Urbaine, $\mathrm{n}^{\circ} 3,365-386$. 
RALLET A, TORRE A, 2001, « Proximité géographique ou proximité organisationelle ? Une analyse spatiale des coopérations technologiques dans les réseaux localisés d'innovation ». Economie Appliquée, tome LIV.

QUERE M, RAVIX JL, 1997, «Relations science-industrie et institutions innovatrices ». Revue d'Economie Industrielle $n^{\circ} 79$.

SAMMUT S, 2000, «Vers une intermédiation effective :petite entreprise et environnement local », Revue internationale des PME, $n^{\circ} 1$.

SAXENIAN AL, 1996, «Beyong boundaries :open labour market and learning in the Sillicon Valley » in MB ATHUER and ROUSSEAU DM (eds). The boundaryness career : a new employment principle for a new organisational area. New York, Oxford University Press.

SHINN T, 1980, «La division du savoir et la spécificité organisationnelle des laboratoires de recherches industrielles en France ». Revue française de sociologie XXI.

STORPER M, 1993, «Regional «worlds »of production : Learning ans innovation in the technology districts in France, Italy and the USA ». Regional Studies 27, pp433-455.

UZZI B, 1997, «Social structure and competition in inter-firm networks: the paradox of embeddedness », Administrative Science Quarterly, 42: 35-67.

VERDIER E, 1999, « Reintroducing public action in the societal analysis ». In MAURICE, SORGUE, Embedding Organizations . John Benjamins Publishing Company; Amsterdam/Philadelphie.

VON HIPPEL E, 1988, Cooperation between rivals: informal knowhow trading, Research Policy

ZIMMERMAN JB, 2000, « De la proximité dans les relations firmes-territoires : nomadisme et ancrage territorial » in J.P. Gilly et A. Torre (Ss la direction de), L'Harmattan, Collection Emploi, Industrie et Territoire. 


\section{Annexe Méthodologique}

Les deux unités de recherche de l'Inria comparées, celles de Sophia Antipolis et celle de Rhône Alpes, en particulier Grenoble, ont été à l'origine d'un nombre significatif de essaimages par rapport à ceux créés nationalement. Elles ont une histoire et un environnement industriel et académiques différents. Elles peuvent être mises en relation avec un « système d'innovation » délimité (contrairement à la Région parisienne).

La répartition des essaimages entre les unités de recherche .

\begin{tabular}{|l|c|c|c|c|c|}
\hline & $\begin{array}{c}\text { Sophia- } \\
\text { Antipolis }\end{array}$ & Rhône-Alpes & $\begin{array}{c}\text { Région } \\
\text { parisienne }\end{array}$ & Lorraine & Bretagne \\
\hline $\begin{array}{l}\text { SO en } \\
\text { activité en } \\
2001\end{array}$ & 7 & 5 & 17 & 5 & 2 \\
\hline
\end{tabular}

Les investigations, support cet article, ont porté sur huit essaimages, 4 pour Sophia Antipolis (SA1, SA2, SA3, SA4), pour Grenoble (G1, G2, G3, G4). Si les essaimages choisis ne sont pas strictement représentatifs des créations d'entreprise de l'Inria dans chacune des deux zones ils illustrent la variété des cas (ancienneté...).

Les investigations ont consisté :

- à recueillir et à analyser la documentation sur les deux zones géographiques prises en compte et sur les conditions de la création des essaimages.

- à réaliser et à analyser des entretiens avec :

- des responsables de la valorisation de l'Inria,

- des chercheurs de l'Inria, membres d'équipes de recherche dont la production de technologie a été à l'origine de la création d'une spin off,

- des créateurs et des salariés des sociétés créées.

Ces entretiens ont été menés sur deux périodes, les créateurs et les salariés des essaimages ont été interviewés une première fois fin 2000, et une seconde fois fin 2001.

Chacune des informations recueillies a été traitée en fonction de la grille d'analyse ci-jointe. 
Les essaimages de l'Inria

\begin{tabular}{|l|c|c|c|c|c|c|c|c|}
\hline & SA1 & SA2 & SA3 & SA4 & G1 & G2 & G3 & G4 \\
\hline $\begin{array}{l}\text { Date de } \\
\text { création }\end{array}$ & 1988 & 1992 & 1998 & 2000 & 1999 & 2000 & 2001 \\
\hline $\begin{array}{l}\text { Nb de } \\
\text { salariés } \\
(\mathbf{2 0 0 0 )}\end{array}$ & 120 & 30 & 20 & 2 puis 30 & 22 puis 32 & 10 puis 14 & 6 vers 10 & 4 \\
\hline CA (2000) & $13 \mathrm{ME}$ & & $25 \mathrm{MF}$ & & $\begin{array}{l}2,4 \mathrm{ME} \\
(2001)\end{array}$ & & Vers $2 \mathrm{ME}$ & $45000 \mathrm{E}$ \\
\hline
\end{tabular}

\section{Activité}

\begin{tabular}{|c|c|c|c|c|c|c|c|c|}
\hline $\begin{array}{l}\text { Segment } \\
\text { d'activités }\end{array}$ & Image spatiale & $\begin{array}{c}\text { Image } \\
\text { médicale }\end{array}$ & $\begin{array}{c}\text { Effets } \\
\text { numériques } \\
\text { pour images } \\
\text { de synthèses }\end{array}$ & $\begin{array}{l}\text { Technologies } \\
\text { Internet }\end{array}$ & $\begin{array}{c}\text { Outils de tests } \\
\text { et de } \\
\text { validation }\end{array}$ & $\begin{array}{c}\text { Editeur de } \\
\text { logiciel }(\mathrm{EL}) \text { : } \\
\text { manufacturier }\end{array}$ & $\begin{array}{c}\text { EL : lien } \\
\text { informatique- } \\
\text { télécoms }\end{array}$ & $\begin{array}{c}\text { EL: } \\
\text { investissemen } \\
\text { ts }\end{array}$ \\
\hline $\begin{array}{l}\text { Type } \\
\text { d'innovation }\end{array}$ & Radicale & Radicale & Incrementale & Incrementale & Incrementale & Incrementale & Incrementale & Incrementale \\
\hline $\begin{array}{l}\text { Type de } \\
\text { marché }\end{array}$ & International & International & International & International & National/local & $\begin{array}{c}\text { Local/Nationa } \\
1\end{array}$ & National/local & International \\
\hline
\end{tabular}

\section{Construction du projet}

\begin{tabular}{|c|c|c|c|c|c|c|c|c|}
\hline $\begin{array}{l}\text { Support de } \\
\text { l'incubation }\end{array}$ & Inria & Inria & $\begin{array}{c}\text { Inria- } \\
\text { Transfert }\end{array}$ & $\begin{array}{c}\text { Inria- } \\
\text { Transfert }\end{array}$ & - & Inria-transfert & $\begin{array}{c}\text { Incubateur } \\
\text { régional }\end{array}$ & Inria-transfert \\
\hline $\begin{array}{l}\text { Type de } \\
\text { fonds } \\
\text { financiers au } \\
\text { Ier amorçage }\end{array}$ & Multiples & $\begin{array}{c}\text { Multiples } \\
\text { dont E-Source }\end{array}$ & E-Source & $\begin{array}{c}\text { Multiples } \\
\text { dont E-Source }\end{array}$ & $\begin{array}{c}\text { Multiples } \\
\text { dont E-Source }\end{array}$ & $\begin{array}{c}\text { Multiples } \\
\text { dont E-Source }\end{array}$ & Multiples & $\begin{array}{c}\text { Multiples } \\
\text { dont E-Source }\end{array}$ \\
\hline
\end{tabular}




\begin{tabular}{|c|c|c|c|c|c|c|c|c|}
\hline $\begin{array}{l}\text { Qualité du } \\
\text { dossier }\end{array}$ & Technologie & Technologie & Technologie & Technologie & Technologie & Technologie & Technologie & Technologie \\
\hline $\begin{array}{l}\text { Intensité du } \\
\text { travail de la } \\
\text { société dans } \\
\text { l'innovation }\end{array}$ & Forte & Forte & Forte & Forte & $\begin{array}{c}\text { Moyen } \\
\text { (spécification } \\
\text { du produit } \\
\text { pour un } \\
\text { marché) }\end{array}$ & $\begin{array}{c}\text { Faible } \\
\text { (spécification } \\
\text { du produit } \\
\text { pour un } \\
\text { marché) }\end{array}$ & $\begin{array}{c}\text { Moyen } \\
\text { (spécification } \\
\text { du produit } \\
\text { pour un } \\
\text { marché) }\end{array}$ & $\begin{array}{c}\text { Moyen } \\
\text { (spécification } \\
\text { du produit } \\
\text { pour un } \\
\text { marché) }\end{array}$ \\
\hline
\end{tabular}

\section{Liens avec l'Inria}

\begin{tabular}{|l|c|c|c|c|c|c|c|c|}
\hline A l'origine & Forts & Forts & Forts & Forts & Moyens & Forts & Moyens & Forts \\
\hline Par la suite & Faibles & Forts & Forts & Faibles & Faibles & - & - & \\
\hline
\end{tabular}

\section{Liens avec l'environnement local}

\begin{tabular}{|l|c|c|c|c|c|c|c|c|}
\hline A l'origine & Moyens & Faibles & Faibles & Faibles & Forts & Forts & Forts & Moyens \\
\hline Par la suite & Faibles & Faibles & Moyens & Moyens & Forts & - & - & \\
\hline
\end{tabular}

\section{Construction de la professionnalité du ou des créateurs}

\begin{tabular}{|c|c|c|c|c|c|c|c|c|}
\hline $\begin{array}{l}\text { Un individu } \\
\text { ou un } \\
\text { collectif }\end{array}$ & Collectif & Individuel & Collectif & Collectif & Collectif & Individuel & Collectif & Collectif \\
\hline Age & Trentaine & Trentaine & Trentaine & $\begin{array}{c}\text { Entre } 25 \text { et } 30 \\
\text { ans }\end{array}$ & Quarantaine & Quarantaine & $\begin{array}{c}\text { Senior (60 } \\
\text { ans) + Jeunes }\end{array}$ & Trentaine \\
\hline $\begin{array}{l}\text { Statut avant } \\
\text { la création }\end{array}$ & $\begin{array}{c}\text { Chercheur } \\
\text { Inria }\end{array}$ & $\begin{array}{l}\text { Chercheur } \\
\text { Inria }\end{array}$ & $\begin{array}{l}\text { Chercheur } \\
\text { Inria et } \\
\text { Ingénieur }\end{array}$ & $\begin{array}{c}\text { Thésards } \\
\text { Inria }\end{array}$ & Hors Inria & $\begin{array}{c}\text { Hors Inria } \\
\text { (CDD Inria) }\end{array}$ & Prof+ thésards & $\begin{array}{c}\text { Chercheur } \\
\text { Inria }\end{array}$ \\
\hline $\begin{array}{l}\text { Formation } \\
\text { initiale }\end{array}$ & $\begin{array}{c}\text { Universitaire/ } \\
\text { Docteur }\end{array}$ & $\begin{array}{c}\text { Universitaire/ } \\
\text { Docteur }\end{array}$ & $\begin{array}{c}\text { Universitaire/ } \\
\text { Docteur et } \\
\text { ingénieur }\end{array}$ & $\begin{array}{c}\text { Universitaire/ } \\
\text { Docteur }\end{array}$ & Ingénieur & Ingénieur & $\begin{array}{c}\text { Ingénieur/doct } \\
\text { eur }\end{array}$ & $\begin{array}{r}\text { Ingénieur/Doc } \\
\text { teur }\end{array}$ \\
\hline
\end{tabular}




\begin{tabular}{|c|c|c|c|c|c|c|c|c|}
\hline $\begin{array}{l}\text { Expérience } \\
\text { professionne } \\
\text { lle }\end{array}$ & $\begin{array}{c}\text { Pas autre qu'à } \\
\text { l'Inria }\end{array}$ & $\begin{array}{c}\text { Pas autre qu'à } \\
\text { l'Inria }\end{array}$ & $\begin{array}{c}\text { Pas autre qu'à } \\
\text { l'Inria et } \\
\text { salarié dans } \\
\text { entreprise }\end{array}$ & $\begin{array}{c}\text { Pas autre qu'à } \\
\text { l'Inria }\end{array}$ & $\begin{array}{c}\text { Dans SO } \\
\text { comme } \\
\text { salarié(techni } \\
\text { que } \\
\text { +management } \\
\text { ) }\end{array}$ & $\begin{array}{c}\text { Dans SO } \\
\text { comme salarié } \\
\text { et comme } \\
\text { créateur }\end{array}$ & $\begin{array}{l}\text { Dans une } \\
\text { Grande } \\
\text { entreprise, } \\
\text { dans le } \\
\text { recherche } \\
\text { académique }\end{array}$ & $\begin{array}{c}\text { Pas autre que } \\
\text { l'Inria }\end{array}$ \\
\hline $\begin{array}{l}\text { Expérience à } \\
\text { l'étranger, } \\
\text { nationale, } \\
\text { locale }\end{array}$ & Aux USA & Aux USA & $\begin{array}{c}\text { Aux USA, au } \\
\text { niveau } \\
\text { national }\end{array}$ & Japon & Locale & Locale & Locale & Aux USA \\
\hline $\begin{array}{l}\text { Liens avec } \\
\text { l'équipe } \\
\text { Inria }\end{array}$ & Forts & Forts & Forts & Forts & Faible & Faible & Fort & Moyen \\
\hline Devenir? & $\begin{array}{c}\text { Retour à } \\
\text { l'Inria ou } \\
\text { chercheur dans } \\
\text { l'entreprise }\end{array}$ & $\begin{array}{l}\text { Retour à } \\
\text { l'Inria ou } \\
\text { chercheur } \\
\text { dans } \\
\text { l'entreprise }\end{array}$ & $\begin{array}{c}\text { PDG ou } \\
\text { responsabilité } \\
\text { locale }\end{array}$ & $\begin{array}{l}\text { Nouveau } \\
\text { créateur }\end{array}$ & PDG & PDG & chercheur & $?$ \\
\hline
\end{tabular}

\section{La transformation de l'essaimage en entreprise.}

\begin{tabular}{|c|c|c|c|c|c|c|c|c|}
\hline Organisée & $\begin{array}{c}\text { Pas à } \\
\text { l'origine }\end{array}$ & $\begin{array}{|ll|}\text { Pas } & \text { à } \\
\text { l'origine } & \\
\end{array}$ & Moyennement & $\begin{array}{ll}\text { Pas } & \mathrm{a} \\
\text { l'origine }\end{array}$ & Dés l'origine & Dés l'origine & Dés l'origine & Dés l'origine \\
\hline $\begin{array}{l}\text { Donne lieu à } \\
\text { des tensions } \\
\text { ou conflits/ } \\
\text { Modification } \\
\text { de trajectoire }\end{array}$ & Oui & Oui & Non & Oui & Non & - & - & - \\
\hline
\end{tabular}

